\title{
Protocolo de acolhimento e atenção para usuários submetidos a endoscopia digestiva alta e seus acompanhantes
}

\author{
Protocol for embracement and attention to users that underwent upper gastrointestinal endoscopy \\ and persons accompanying them
}

Protocolo para el acogimiento y la atención a los usuarios que se sometieron a una endoscopia digestiva alta y sus acompañantes

\section{Ilza Schmidt de Brito Selhorst', Maria Bettina Camargo Bub', Juliana Balbinot Reis Girondi'} 'Universidade Federal de Santa Catarina, Departamento de Enfermagem, Programa de Pós-Graduação em Enfermagem.
Florianópolis-SC, Brasil.

Submissão: 25-01-2013 Aprovação: 19-06-2014

\section{RESUMO}

Pesquisa quanti-qualitativa com o objetivo de construir um protocolo de acolhimento e atendimento para os usuários submetidos à Endoscopia Digestiva Alta e seus acompanhantes, num Centro Endoscópico de um hospital do sul do país. Esse protocolo foi elaborado através da construção coletiva de um grupo de enfermeiras, e validado posteriormente por três peritos. Os referenciais teóricos de sustentação da proposta foram a Política Nacional de Humanização e a Teoria das Necessidades Humanas Básicas de Wanda Horta. A institucionalização do protocolo possibilitou o acolhimento e o atendimento ao usuário submetido ao procedimento endoscópico e seu acompanhante nos períodos pré, intra e pós-exame. Propiciou ainda a instrumentalização e organização do processo de trabalho da equipe de enfermagem garantindo, assim, a segurança do paciente durante todo o processo.

Descritores: Protocolo; Assistência de Enfermagem; Acolhimento; Endoscopia.

\section{ABSTRACT}

This is a quantitative and qualitative research aimed at building a Welcome Protocol for users and their companions referred to the Endoscopic Centre at the University Hospital Dr. Polydoro Ernani São Thiago, at the Federal University of Santa Catarina (UFSC), and submitted to Upper Digestive Endoscopy. The research was elaborated in two stages. The first stage was the building of a collective protocol with a group of nurses; the second stage was the sending of this material, organized as a protocol, for validation by the three experts. The study's supporting theoretical background was the National Humanization Policy and the Basic Human Needs theory. The protocol built aims at detailing the procedures conducted in the pre, trans and post-endoscopy exam stages, permitting the organization of the service and the instrumentalization of the professional team and, thus, ensuring a safer welcome and service to the user and his/her companion.

Key words: Protocol; Nursing Care; User Embracement; Endoscopy.

\section{RESUMEN}

Trata-se de una investigación cuantitativa y cualitativa con el objetivo de construir un protocolo de acogimiento para los usuarios - y sus compañeros - encaminados al Centro Endoscópico del Hospital Universitario Dr. Polydoro Ernani São Thiago de la Universidad Federal de Santa Catarina, para ser sometidos a Endoscopia Digestiva Alta. Fue elaborada en dos fases: la primera fue la construcción colectiva del protocolo con un grupo de enfermeras; la segunda fue el envío de ese material organizado bajo forma de protocolo, para validación junto a los tres peritos. El referencial teórico de sustentación de ese estudio fue la Política Nacional de Humanización y la teoría de las Necesidades Humanas Básicas. El protocolo construido tiene la pretensión de explicitar los procedimientos ejecutados en el período pre, trans y pos-examen de endoscopia, posibilitando la organización del servicio y la instrumentalización del equipo de profesionales garantizando, de esa manera, un acogimiento y atendimiento más seguro para el usuario y su compañero.

Palabras clave: Protocolo; Atención de Enfermería; Acogimiento; Endoscopia.

\section{AUTOR CORRESPONDENTE Juliana Balbinot Reis Girondi E-mail: juliana.balbinot@ufsc.br}




\section{INTRODUÇÃO}

A Endoscopia Digestiva Alta consiste em um procedimento invasivo para inspeção de órgãos e cavidades do corpo, por meio de um endoscópio, capaz de gerar um grau de incômodo, de acordo com a tolerância da pessoa ${ }^{(1)}$. Tem finalidades diagnósticas e terapêuticas. A realização desse exame é uma prerrogativa médica, porém demanda atenção e atuação da Enfermagem em todos os momentos. Ou seja, desde o momento do preparo de materiais, instrumentais, equipamentos e ambiente, perpassando pelo acolhimento do usuário e seu acompanhante, até o momento da realização do exame e das práticas de educação em saúde e em serviço.

Fatores como a aquisição de equipamentos mais modernos e complexos, o conhecimento acerca dos problemas ligados à desinfecção passíveis de serem provocadas por aparelhos endoscópicos, as exigências legais decorrentes destes riscos, as mudanças no perfil do usuário do serviço, que aumenta em termos de complexidade e gravidade, levaram a necessidade de planejar a assistência de enfermagem de forma mais individualizada e segura.

Nesse contexto emergiu a proposta de construção de um protocolo de acolhimento e atendimento para nortear as ações do enfermeiro e da equipe de enfermagem atuantes num setor de exames endoscópicos.

Um protocolo de atendimento é um conjunto de dados que permite direcionar o trabalho e registrar oficialmente os cuidados executados para a resolução ou prevenção de um problema. A implantação de protocolos proporciona efetivamente um instrumento de mudanças e de aumento de capacidade crítica dos profissionais, melhorando o desempenho e a postura individual e coletiva, gerando um trabalho capaz de produzir mais saúde ${ }^{(2)}$.

Quando pensamos na concretização de um cuidado humanizado(3-5), acolhedor e seguro para o serviço de saúde devemos considerar as particularidades e complexidades do indivíduo que nos procura, principalmente quando decidimos adotar novas formas de organização da estrutura, dos processos de trabalho existentes e de uma prática assistencial diferenciada. Tais ações são desenvolvidas desde o momento da recepção do usuário no serviço, onde o mesmo é encaminhado para uma consulta de enfermagem. Nessa consulta, o enfermeiro realiza o Histórico de Enfermagem, através da entrevista, anamnese e exame físico; desenvolve ações educativas e de orientações, além da prescrição de enfermagem, de acordo com as necessidades e peculiaridades de cada usuário.

Dessa forma ao construirmos um protocolo de acolhimento e atendimento contamos com os resultados do estudo realizado anteriormente neste hospital de ensino ${ }^{(6)}$, com o usuário de saúde submetido à Endoscopia Digestiva Alta com o fim de diagnóstico e seu acompanhante, que permitiu elaborar o perfil e as suas percepções a respeito do cuidado. Constatamos também a importância de implantar o acolhimento ${ }^{(7)}$ como uma prática de saúde, levando em conta quem são os envolvidos na assistência.

O protocolo de acolhimento e atendimento construído por sugestão dos usuários de saúde e seus acompanhantes contemplou as atividades assistenciais e gerenciais que a enfermeira e sua equipe deveriam executar no período pré, trans e pós-exame de endoscopia digestiva alta, além de ter permitido a instrumentalização e motivação da equipe de profissionais de enfermagem do Centro Endoscópico, para uma prática crítico-reflexiva e inovadora.

Portanto, esta pesquisa teve por objetivo construir um protocolo de acolhimento e atendimento para os usuários submetidos à Endoscopia Digestiva Alta e seu acompanhante num Centro Endoscópico de um hospital do sul do país. O protocolo norteou as ações da equipe de enfermagem, quanto ao acolhimento e atendimento do usuário de saúde e seu acompanhante desde o momento de chegada até sua liberação do serviço. Ainda propiciou a organização do processo de trabaIho e instrumentalização da equipe de enfermagem.

\section{MÉTODOS}

O estudo foi realizado no Centro Endoscópico do Hospital Universitário Dr. Polydoro Ernani São Thiago, da Universidade Federal de Santa Catarina, uma instituição de ensino e saúde ligada ao Ministério da Educação. Este hospital iniciou suas atividades em 1980, tem porte de complexidade média e serve de campo de aprendizagem para os cursos de Ciências da Saúde, Serviço Social, Psicologia, dentre outros. Atende a demanda dos serviços de ambulatório, emergência adulto, emergência pediátrica e ginecológica. Possui serviços de internação com 261 leitos ativos e disponíveis nas áreas de clínicas médicas, cirúrgicas, pediátrica, obstetrícia, Unidade de Tratamento Intensivo e Unidade de Tratamento Dialítico.

A construção do protocolo de acolhimento e atendimento foi realizada com a pesquisadora e as três enfermeiras que atuam no Centro Endoscópico. Para esta fase foram realizados três encontros com o grupo de enfermeiras, com duração aproximada de duas horas. O horário e os dias das reuniões foram agendados conforme consenso do grupo, nos meses de julho e agosto de 2011.

As atividades foram organizadas da seguinte forma: no primeiro encontro foi feito uma descrição da rotina já existente no serviço em relação aos procedimentos realizados pela equipe de enfermagem e a apresentação do perfil e da percepção do usuário submetido à endoscopia digestiva alta e seu acompanhante $^{(6)}$. No segundo encontro foi realizada uma análise do perfil e percepções dos usuários e seus acompanhantes, a elaboração dos indicativos que seriam usados como subsídios para a construção do protocolo e a elaboração das principais intervenções de enfermagem. Estes constructos foram enviados via correio eletrônico para a pesquisadora. No último encontro foram identificados os pontos de consenso e dissenso, registradas as decisões e propostas apresentadas, esboçado em linhas gerais como seria composto o protocolo. Posteriormente, a pesquisadora responsável elaborou a versão preliminar do protocolo. Esta etapa de organização do protocolo ocorreu no mês de setembro de 2011.

A etapa final da pesquisa teve como propósito validar o protocolo construído mediante parecer dos peritos e ocorreu no mês de outubro de 2011. Participaram dessa etapa duas enfermeiras não vinculadas à instituição, que tinham vasta 
experiência na área de endoscopia, organização de serviços e protocolos; e um médico docente que tinha vínculo empregatício com a instituição e experiência na área. Cada perito emitiu seu relatório final com parecer favorável validando a proposta.

A pesquisa foi avaliada e aprovada pelo Comitê de Ética em Pesquisa com Seres Humanos da Universidade Federal de Santa Catarina pelo protocolo n. 1857, FR 409026.

\section{RESULTADOS E DISCUSSÃO}

Na elaboração do protocolo o grupo procurou desenvolver um instrumento prático, adequado à instituição, de linguagem acessível, disponível para o conhecimento de toda a equipe de saúde e que pudesse ser atualizado sempre que houvesse a necessidade para tal. O grupo tinha clareza que o procedimento endoscópico é uma intervenção que exige uma atuação multiprofissional, especialmente por parte da equipe médica e de enfermagem. Especialmente em virtude desse fato persistia a consciência da necessidade de estabelecer um protocolo de acolhimento e atendimento com indicações das responsabilidades a serem assumidas e a competências técnicas da enfermagem nesse processo.

O maior desafio para a construção do protocolo foi contrapor a maneira mais adequada de trabalhar com a incorporação dessa nova tecnologia na nossa prática assistencial, aliando o conhecimento técnico-científico emergente, com os já existentes e seguidos pelos profissionais que lá atuavam levando. Além de considerar o perfil e as percepções dos usuários de saúde e acompanhantes que frequentavam esse serviço ${ }^{(8-9)}$.

Para fins didáticos e facilitar a compreensão da proposta, as enfermeiras em conjunto com a pesquisadora optaram por estruturar as atividades de enfermagem de acordo com: 1) o período de realização do procedimento (pré, trans e pós-exame); 2) o fluxo do usuário e seu acompanhante no Centro Endoscópico. O protocolo, construído pelas enfermeiras do serviço e validado pelos experts da área, descreve sistematicamente e sucintamente as atribuições e competências de cada membro da equipe de enfermagem.

Quadro 1 - Protocolo do período que antecede o exame de Endoscopia Digestiva Alta

\begin{tabular}{|c|c|}
\hline \multicolumn{2}{|c|}{$\begin{array}{l}\text { Período que antecede o exame de Endoscopia Digestiva Alta: Compreende o acolhimento e os cuidados de enfermagem dispensados ao usuário } \\
\text { e seu acompanhante no serviço, a partir do momento da sua inclusão até o seu preparo para o exame. }\end{array}$} \\
\hline \multicolumn{2}{|r|}{ Atividades Assistenciais } \\
\hline & Cuidados de Enfermagem \\
\hline \multirow[b]{2}{*}{$\begin{array}{c}\text { Área do } \\
\text { consultório } \\
\text { Profissional apto } \\
\text { para atuar nesta } \\
\text { área: enfermeiro. }\end{array}$} & $\begin{array}{l}\text { 1. Enfermeiro do Centro Endoscópio chama o usuário e seu acompanhante seguindo os critérios de atendimento, para o } \\
\text { consultório de enfermagem onde será realizada a consulta de enfermagem. }\end{array}$ \\
\hline & $\begin{array}{l}\text { 2. Enfermeiro procederá: } \\
\text { - Conferir presença do acompanhante e se o usuário está } 12 \text { horas de jejum. Caso não tiver cumprido o tempo de jejum, } \\
\text { será informado ao médico endoscopista que se responsabilizará pela decisão da transferência ou não da data do exame. } \\
\text { Caso indicado será reorientado sobre a necessidade do cuidado e remarcado novo dia para fazer o exame. } \\
\text { - Investigar a presença de acompanhante com o usuário. Para a falta do acompanhante serão dadas as opções de entrar } \\
\text { em contato telefônico com um familiar para vir até a instituição antes de iniciar o exame OU fazer o procedimento sem } \\
\text { sedação OU retornar para unidade básica de saúde e remarcar o exame. } \\
\text { - Solicitar que ao usuário que faça a leitura, preencha e assine o termo de consentimento(10) para realização do exame. } \\
\text { Caso ele declare não ter condições para tal solicitará que o acompanhante faça a leitura ou ele próprio fará este papel. } \\
\text { No final da leitura o usuário se concordar com o procedimento deverá assinar ou colocar sua digital e uma testemunha } \\
\text { deverá assinar o documento. } \\
\text { - Coletar os dados para preenchimento do Histórico de Enfermagem. O modelo utilizado como Histórico de Enfermagem } \\
\text { é o baseado nas Necessidades Humanas Básicas de Horta padronizado na instituição desde } 1980 \text { (inclui Anamnese e } \\
\text { exame físico). O instrumento permite coletar dados essenciais para posteriormente definir o plano de cuidados antes, } \\
\text { durante e após o procedimento endoscópico. }\end{array}$ \\
\hline \multirow{2}{*}{$\begin{array}{l}\text { Área de preparo } \\
\text { pré-exame } \\
\text { Profissional apto } \\
\text { para atuar nesta } \\
\text { área: técnico } \\
\text { ou auxiliar } \\
\text { de enfermagem. }\end{array}$} & $\begin{array}{l}\text { 3. Enfermeiro deverá: } \\
\text { - Orientar o usuário e seu acompanhante sobre o procedimento (a equipe que o assistirá durante o procedimento, os } \\
\text { cuidados necessários antes/durante e após o procedimento, o que é o exame, o que é a sedação, como o usuário deve se } \\
\text { comportar durante o procedimento e como é a recuperação pós-exame). } \\
\text { - Entregar o Folder Explicativo, que contempla todas estas informações descritas anteriormente, caso este não tenha sido } \\
\text { entregue no momento da marcação exame. } \\
\text { - Proporcionar espaço para questionamentos e esclarecimento de dúvidas. }\end{array}$ \\
\hline & $\begin{array}{l}\text { 4. O enfermeiro deverá: } \\
\text { - Encaminhar o usuário até a sala de preparo pré-exame. } \\
\text { - Solicitar que o acompanhante permaneça na sala de recepção, com os pertences pessoais do usuário. }\end{array}$ \\
\hline $\begin{array}{l}\text { Supervisão: } \\
\text { Enfermeiro }\end{array}$ & $\begin{array}{l}\text { 5. Nos casos dos usuários internados na instituição previamente à data do exame o enfermeiro deverá: } \\
\text { - Realizar uma visita pré-exame. } \\
\text { - Realizar o Histórico de Enfermagem. } \\
\text { - Entregar o Termo de consentimento livre e esclarecido para a autorização da realização do procedimento. } \\
\text { - Entregar o Folder explicativo, esclarecer dúvidas e fazer as orientações necessárias de acordo com as necessidades e } \\
\text { especificidades de cada usuário. }\end{array}$ \\
\hline
\end{tabular}




\begin{tabular}{|c|c|}
\hline & 6. O técnico de enfermagem receberá o usuário já orientado previamente na consulta de enfermagem. \\
\hline $\begin{array}{c}\text { Área de preparo } \\
\text { pré-exame }\end{array}$ & $\begin{array}{l}\text { 7. O técnico de enfermagem responsável pela área de preparo pré-exame, antes de iniciar suas atividades, deverá: } \\
\text { - Organizar o ambiente e realizar o checklist. O checklist da área é um instrumento que auxiliará no sucesso na realização } \\
\text { de suas atividades rotineiras ou se alguma intercorrência ocorrer. }\end{array}$ \\
\hline $\begin{array}{l}\text { Profissional apto } \\
\text { para atuar nesta } \\
\text { área: técnico } \\
\text { ou auxiliar } \\
\text { de enfermagem. }\end{array}$ & $\begin{array}{l}\text { 8. O técnico de enfermagem recepcionará o usuário e iniciará os cuidados de enfermagem relacionados ao preparo pré- } \\
\text { procedimento: } \\
\text { - Acomodar o usuário em poltrona, verificará se foram retirados óculos, bonés, prótese dentária, aparelho móvel e entregues } \\
\text { ao acompanhante. Caso isso não tenha ocorrido, deverá proceder à tarefa de colocar em embalagem identificada e } \\
\text { guardar em local próprio. } \\
\text { - Puncionar acesso venoso periférico, preferencialmente em membro superior direito. } \\
\text { - Administrar dose de Dimeticona via oral conforme prescrito pelo médico endoscopista. } \\
\text { - Fazer os registros no espaço destinado às observações complementares, que consta do histórico de enfermagem. } \\
\text { - Aferir os sinais vitais, verificar glicemia capilar e outros procedimentos conforme necessidade. } \\
\text { - Encaminhar o usuário para sala de procedimento. }\end{array}$ \\
\hline
\end{tabular}

\section{Quadro 2 - Protocolo do período durante o exame de Endoscopia Digestiva Alta}

Período durante o exame de Endoscopia Digestiva Alta: Com o usuário acolhido e preparado pela Enfermagem para ser submetido à Endoscopia, ele é encaminhado para a área de exame. Nesta parte do protocolo estão descritos todos os cuidados dispensados desde o momento que ele é recepcionado, passando pelo procedimento, seu transporte para a área de recuperação até o preparo da sala para o próximo exame.

1. O funcionário responsável pela sala recepcionará o usuário, se identificará e se colocará disponível para solucionar as dúvidas que persistirem.

2. O técnico de enfermagem responsável antes de iniciar as atividades deverá:

- Organizar a sala e checar todos os equipamentos endoscópicos, instrumentais, materiais e acessórios que poderão ser utilizados nos procedimentos.

- Preparar na bancada próxima ao local onde o usuário será atendido o Kit para sedação, as drogas antagonistas, drogas para hemostasia, cateteres para escleroterapia, pinças de biópsia, pinças para retirada de corpo estranho, alça de polipectomia, kit de ligadura elástica e dilatadores esofágicos.

- Realizar o checklist onde deverá testar e verificar a funcionalidade dos equipamentos de videoendoscopia; sistema de fornecimento de oxigênio a 100\%; vácuo para aspirar secreções, sangue e coágulos; oximetria de pulso.

- Colocar próximo à torre endoscópica: kit de teste rápido de Urease, frascos com formol para biópsia e corantes para cromoscopia.

3. O técnico de enfermagem após ter se identificado deverá:

- Conferir novamente a identificação do usuário com sua documentação, quais sejam: autorização do sistema de regulação para realização de exames de usuários externos, pedido médico do exame, termo de consentimento livre e esclarecido assinado, resultados de exames anteriores (quando houver) e histórico de enfermagem.

Área de exame de Endoscopia

Profissional apto para atuar nesta área: técnico ou auxiliar de enfermagem.

Supervisão: Enfermeiro
4. O técnico de enfermagem considerando o usuário apto a submeter-se ao exame irá:

- Posicionar na maca o usuário em decúbito lateral esquerdo, cabeça sob o travesseiro com flexão cervical anterior, toalha próxima à face.

- Instilar medicação à base de lidocaína 10\% em orofaringe e ajustar o bocal em cavidade oral.

- Conectar o oxímetro em quirodáctilo, de preferência na mão direita.

- Confirmar com o médico responsável a dose de medicamento analgésico e sedativo e administrará conforme prescrito ${ }^{(11)}$.

- Instalar oxigenoterapia conforme prescrição médica.

- Posicionar-se no lado esquerdo da maca próximo á cabeça do usuário segurando o bocal e restringindo-o mecanicamente. O outro profissional da enfermagem ficará do lado direito da maca, próximo ao endoscopista para auxiliá-lo no manuseio dos acessórios, equipamentos e na realização de procedimentos.

5. Após a realização do procedimento o profissional de enfermagem deverá:

- Observar frequência cardíaca e oxímetria, informando ao médico ou enfermeiro qualquer alteração.

- Manter o usuário em decúbito lateral esquerdo.

- Levantar grades da maca.

- Retirar o oxímetro e o bocal.

- Encaminhar o usuário até a área de preparo/recuperação.

- Dar os devidos encaminhamentos aos materiais, equipamentos e acessórios utilizados e às amostras que foram coletadas para exames.

6. O funcionário responsável pela área de exame ao entregar o usuário para o profissional que irá recebê-lo na área de recuperação pós-exame deverá:

- Informar o nome do usuário que foi submetido ao procedimento, tipo de procedimento realizado, tipo de sedativo e dose aplicada, intercorrências durante o exame, informações sobre os sinais vitais, se houve necessidade de oxigenoterapia, sinais de vasoconstricção periférica e outros.

- Proceder aos registros de enfermagem no impresso Histórico de enfermagem. 


\begin{tabular}{|c|c|}
\hline $\begin{array}{l}\text { Área de exame } \\
\text { de Endoscopia } \\
\text { Profissional apto } \\
\text { para atuar nesta } \\
\text { área: técnico } \\
\text { ou auxiliar de } \\
\text { enfermagem. }\end{array}$ & $\begin{array}{l}\text { 7. Na área de endoscopia após ser realizado o último exame o profissional deverá proceder à limpeza e organização da } \\
\text { sala da seguinte forma: } \\
\text { - Identificar frascos de biópsia ou outros exames, colocar em local próprio e encaminhar ao serviço de patologia protocolado. } \\
\text { - Descartar bocal e aplicador de solução de lidocaína em recipiente próprio e transportar para sala de limpeza e desinfecção } \\
\text { - de materiais. } \\
\text { - Descartar sobras de medicações em recipiente próprio. } \\
\text { - Proceder à limpeza da torre de endoscopia com álcool } 70 \% \text {. } \\
\text { - Repor material como: seringa, agulha, gaze, acessórios, cateter de oxigênio, umidificador e outros. } \\
\text { - Trocar vidro de aspiração e encaminhar até a sala de limpeza de materiais, repondo frasco limpo. } \\
\text { - Fazer a pré-limpeza imediata do endoscópio com gaze e aspiração de solução enzimática. } \\
\text { - Transportar o tubo endoscópico para área de limpeza e desinfecção de materiais em contêiner }{ }^{(12) .} \text {. } \\
\text { - Fazer teste de infiltração diariamente, seguindo as orientações do fabricante do endoscópio. }\end{array}$ \\
\hline
\end{tabular}

Quadro 3 - Protocolo do período após o exame de Endoscopia Digestiva Alta

Período após o exame de Endoscopia Digestiva Alta: Nesta parte temos o detalhamento dos cuidados dispensados na recuperação do usuário a partir de sua chegada à área de preparo pré-exame e recuperação pós-exame até a saída do Centro Endoscópico com o seu acompanhante. Constam também as atividades na área de limpeza e desinfecção de equipamentos.

\begin{tabular}{|c|c|}
\hline $\begin{array}{l}\text { Área de } \\
\text { recuperação } \\
\text { pós-exame }\end{array}$ & $\begin{array}{l}\text { 1. O funcionário deverá monitorar o usuário durante o repouso e seguir as seguintes recomendações: } \\
\text { - Manter o posicionamento do usuário em decúbito lateral esquerdo, com a cabeça elevada. } \\
\text { - Manter oximetria, verificar pressão arterial e glicemia capilar. } \\
\text { - Observar sinais de reação alérgica, sangramentos, dor, desconforto abdominal, náusea, vomito. } \\
\text { - Manter usuário aquecido e confortável. } \\
\text { - Instalar fluidoterapia e administrar medicações conforme prescrição médica. } \\
\text { - Avaliar o nível de consciência e atividade motora sob comando, seguindo o estabelecido no impresso Histórico de Enfermagem. }\end{array}$ \\
\hline \multirow[t]{2}{*}{$\begin{array}{l}\text { Profissional apto } \\
\text { para atuar nesta } \\
\text { área: técnico } \\
\text { ou auxiliar } \\
\text { de enfermagem. }\end{array}$} & $\begin{array}{l}\text { 2. O técnico de enfermagem responsável por esta área deverá: } \\
\text { - Seguir os critérios para alta do usuário após sedação, que consta do impresso Histórico de enfermagem e fará uma nova } \\
\text { estimativa do nível de consciência do usuário. } \\
\text { - Informará à enfermeira que irá avaliar as condições de alta e liberação do usuário. }\end{array}$ \\
\hline & $\begin{array}{l}\text { 3. O técnico de enfermagem acompanhará o usuário até a área de recepção e na presença do acompanhante deverá entregar o } \\
\text { laudo do exame, autorização para pegar o resultado da biópsia (caso tenha sido realizada), atestado médico, orientações por } \\
\text { escrito e informar quanto à necessidade de atenção para intercorrências como febre, sangramentos e queixas álgicas. Deverá } \\
\text { solicitar que procure a Emergência do hospital se observar alguma destas alterações e esclarecer as dúvidas se existirem. } \\
\text { - O enfermeiro deverá ser responsável por encaminhar o usuário até o acompanhante na recepção, nos casos de } \\
\text { intercorrência durante o procedimento, ou da necessidade de uma orientação específica. Prestar informação verbal e por } \\
\text { escrito garantindo a compreensão do acompanhante. }\end{array}$ \\
\hline $\begin{array}{l}\text { Profissional apto } \\
\text { para atuar nesta } \\
\text { área: técnico } \\
\text { ou auxiliar } \\
\text { de enfermagem. }\end{array}$ & $\begin{array}{l}\text { 1. O protocolo de limpeza e desinfecção dos endoscópios segue as seguintes etapas: } \\
\text { - Procedimento inicial de limpeza, limpeza mecânica de forma manual, - limpeza mecânica e manual com escovação dos } \\
\text { canais, - retirada de resíduos dos canais, - limpeza com esponja por fricção, - limpeza manual das bordas dos canais, - } \\
\text { procedimento final da limpeza com enxague utilizando os acessórios do aparelho fornecidos pelo fabricante e secagem } \\
\text { interna com jato de ar, - limpeza por escovação das válvulas, -limpeza da garrafa de água, -preparo para desinfecção com } \\
\text { a colocação do aparelho dentro da cuba com produto desinfetante no tempo preconizado pelo fabricante do produto, } \\
\text {-após o término da desinfecção colocar o endoscópio em outra cuba e fazer o enxágue interno e externo, - secagem } \\
\text { interna e externa do aparelho com o ar comprimido e transporte para sala de exame ou armazenamento em local } \\
\text { apropriado. }\end{array}$ \\
\hline
\end{tabular}

\section{CONCLUSÕES}

A vivência em elaborar um protocolo levou o grupo de enfermeiros a desenvolver um pensamento mais crítico ao rever a sua forma de atuação, seus conhecimentos sobre sua prática, de como sua equipe realiza o trabalho e a responsabilidade de cada profissional de enfermagem ao desenvolver as atividades.

As contribuições para a construção do protocolo de acoIhimento e atendimento foram vastas, uma vez que repercutiu significativamente em nossa prática cotidiana e no processo de trabalho da equipe de enfermagem. Evidenciou-se que, ao repensarmos nossa prática, além de compartilharmos nossos conhecimentos assumimos responsabilidades com a nossa prática, com o usuário do nosso serviço e seu acompanhante.

A validação efetivada por peritos externos à instituição possibilitou uma compartilhar de informações e conhecimentos com outras realidades, uma visão de quem não está envolvido diretamente com o processo de construção. Mostrou que o protocolo permite inclusões e adaptações e que é necessário prever revisões periódicas para acompanhar as atualizações das novas tecnologias de cuidado nessa área.

Por fim, espera-se com esse protocolo promover um adequado acolhimento e atendimento mais seguro para o usuário e seu acompanhante e, quiçá que o mesmo possa servir de subsídio para outros serviços que vivenciam semelhante realidade. 


\section{REFERÊNCIAS}

1. Silva MG. Enfermagem em endoscopia: digestiva e respiratória. São Paulo (SP): Atheneu; 2010.

2. Werneck MAF, Faria HP, Campos KFC. Protocolos de cuidado à saúde e de organização do serviço. Belo Horizonte (BH): Coopmed; 2009. Co-publicado por Nescon/ UFMG.

3. Boareto C. Humanização da assistência hospitalar: o dia a dia da prática dos serviços. Ciênc Saúde Coletiva. 2004;19(1):15-29.

4. Ministério da Saúde (BR). HumanizaSUS: Política Nacional de Humanização: a humanização como eixo norteador das Práticas de Atenção e Gestão em todas as instâncias do SUS [Internet]. Brasília (DF): Ministério da Saúde; 2004 [acesso em 25 de janeiro de 2013]. Disponível em: http://bvsms.saude.gov.br/bvs/publicacoes/humanizasus_2004.pdf

5. Campos GWS. Humanização na saúde: um projeto em defesa da vida? Interface Comum Saúde Educ. 2005;9(17):389-406.

6. Selhorst ISB. Protocolo de acolhimento para usuários submetidos à endoscopia digestiva alta e seus acompanhantes [dissertação]. Florianópolis (SC): Universidade Federal de Santa Catarina; 2011.

7. Ministério da Saúde (BR). Acolhimento e classificação de risco nos serviços de urgência [Internet]. Brasília (DF): Ministério da Saúde; 2009 [acesso em 05 de junho de 2010]. Disponível em: http://bvsms.saude.gov.br/bvs/publicacoes/acolhimento_classificaao_risco_servico_urgencia.pdf
8. Magnago TSBS, Rosa TP, Tavares JP, Lima SBS, Schimidt MD, Silva RM. Perfil dos pacientes atendidos na sala de emergência do pronto socorro de um hospital universitário. Rev Enferm UFSM [Internet]. 2011 [acesso em 05 de junho de 2010];1(1):51-60. Disponível em: http:// cascavel.cpd.ufsm.br/revistas/ojs-2.2.2/index.php/reufsm/ article/view/2090/1511

9. Oliveira PS, Nóbrega MML, Silva ATMC, Ferreira-Filha MO. Comunicação terapêutica em enfermagem revelada nos depoimentos de pacientes internados em centro de terapia intensiva. Rev Eletrônica Enferm [Internet]. 2005 [acesso em 25 de janeiro de 2013];7(1):54-63. Disponível em: http://www.revistas.ufg.br/index.php/fen/article/ view/861/1035

10. Oliveira VL, Pimentel D, Vieira MJ. O uso do termo de consentimento livre e esclarecido na prática médica. Rev Bioét. 2010;18(3):705-24.

11. López Roses L. Sedation/analgesia guidelines for endoscopy. Rev Esp Enferms Dig [Internet]. 2006 [cited 2013 January 25];98(9):685-92. Available from: http:// www.grupoaran.com/mrmUpdate/lecturaPDFfromXML. asp $?$ IdArt $=458293 \&$ TO $=$ RVN\&Eng $=0$

12. Agência Nacional de Vigilância Sanitária (BR); Sociedade Brasileira de Enfermagem em Endoscopia Gastrointestinal (BR). Manual de limpeza e desinfecção de aparelhos endoscópicos [Internet]. [S.I]: ANVISA, SOBEEG; 2006 [acesso em 25 de janeiro de 2013]. Disponível em: http://www.anvisa.gov.br/servicosaude/manuais/sobeeg_manual.pdf 